\title{
Gracilis Muscular Flap for Large Urethral Defect
}

\author{
Yuichiro Yoshioka, Shigeaki Moriura, Hiroshi Yuba, Atsushi Hirano, Ryohei Hattori \\ Department of Surgery and Urology, Yachiyo Hospital, Anjo, Japan \\ E-mail:moriura@yachiyo-hosp.or.jp
}

Received July 22, 2011; revised October 13, 2011; accepted October 28, 2011

\begin{abstract}
We report the use of Gracilis muscle to repair a large urethral defect. A 57-year-old-man with rectal cancer underwent abdominoperineal resection including part of the prostate and seminal vesicle. Soon after surgery, he presented with massive urinary leakage from the prostatic urethra. Conservative treatment for one month failed. The defect of the prostatic urethra, measuring $2.5 \mathrm{~cm}$ in diameter, was closed with the right gracilis muscular flap. About five years and 6 months after surgery, the patient can void spontaneously without incontinence. Cystoscopy demonstrated good epithelization of the reconstructed urethra without stenosis. The gracilis muscular flap was easily available and useful for closure of a large urethral defect.
\end{abstract}

Keywords: Urethral Injury, Reconstruction

\section{Introduction}

In a case of rectal cancer with a limited invasion to the prostate, partial resection of the prostate should be tried to avoid pelvic exenteration. However, there is a risk of urethral injury, which may cause urethral fistula. We describe a case of a postoperative urethral rupture in which a gracilis muscular flap was useful for repair.

\section{A Case Report}

A 57-year-old man was referred to our hospital with a diagnosis of lower rectal cancer. Abdominoperineal resection was performed. We resected the posterior part of the prostate and right seminal vesicle because of the tumor invasion to the prostate. There was no apparent injury to the urethra. The resected prostate was round and measured $2 \mathrm{~cm}$ in diameter, 2 - $4 \mathrm{~mm}$ in thickness. Soon after surgery, there was significant urinary discharge from the pelvic drain. Cystography demonstrated urinary leakage from the prostatic urethra to the pelvic cavity (Figure 1). The balloon of the catheter was seen in the pelvic cavity. We changed the urethral catheter to that with a larger balloon and tried to close the fistula conservatively. One month later, there was no improvement of leakage or change in fistula size. We decided to close the fistula surgically.

The patient was placed in the lithotomy position and we re-opened the perineal wound. There were no signs of infection in the pelvic cavity. The size of the urethral defect was about $2.5 \mathrm{~cm}$ in diameter (Figure 2). Two thirds of the muscular body of the gracilis muscle was separated from the proximal end and the vascular pedicle located at the proximal part was preserved. The gracilis muscular flap was transferred to the pelvic cavity through a subcutaneous route. The urethral defect was closed using the middle part of the flap with absorbable sutures as if the muscle were a patch. The pelvic cavity was filled with the distal part of the flap (Figure 3) Suction drains were placed in the pelvic cavity and the donor site and a cystostomy was established. Postoperatively, there was slight urinary leakage, which was treated conservatively. Cystography 4 weeks postoperatively did not demonstrate any leakage (Figure 4). The indwelling urethral catheter was removed 7 weeks postoperatively and the cystostomy catheter was removed 12 weeks after surgery. The patient complained of occasional urinary incontinence, which improved gradually, and there was almost no incontinence 14 months postoperatively. Histological examination demonstrated that rectal cancer had invaded the prostate. About five years and 6 months after the surgery, cystoscopy demonstrated good epithelization of the reconstructed urethra without stenosis. The patient can void spontaneously without incontinence and there are no symptoms of urethral stenosis.

\section{Discussion}

In the current case, urinary leakage manifested in the early postoperative period. The cystography demonstrated that 


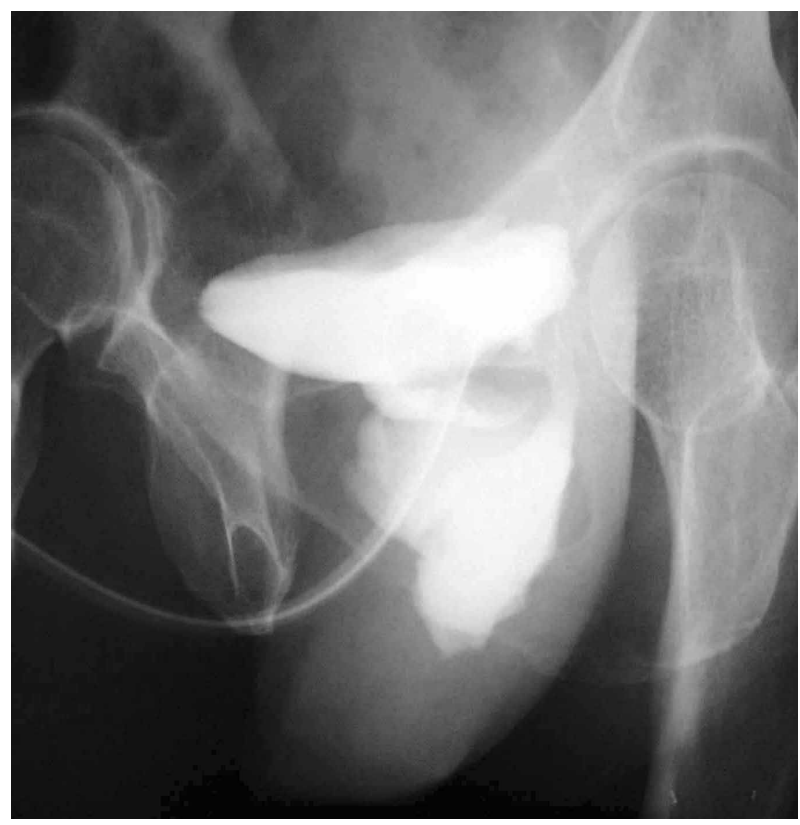

Figure 1. Cystography shows urinary leakage from the prostatic urethra to the pelvic cavity. The balloon of the catheter is seen in the pelvic cavity.

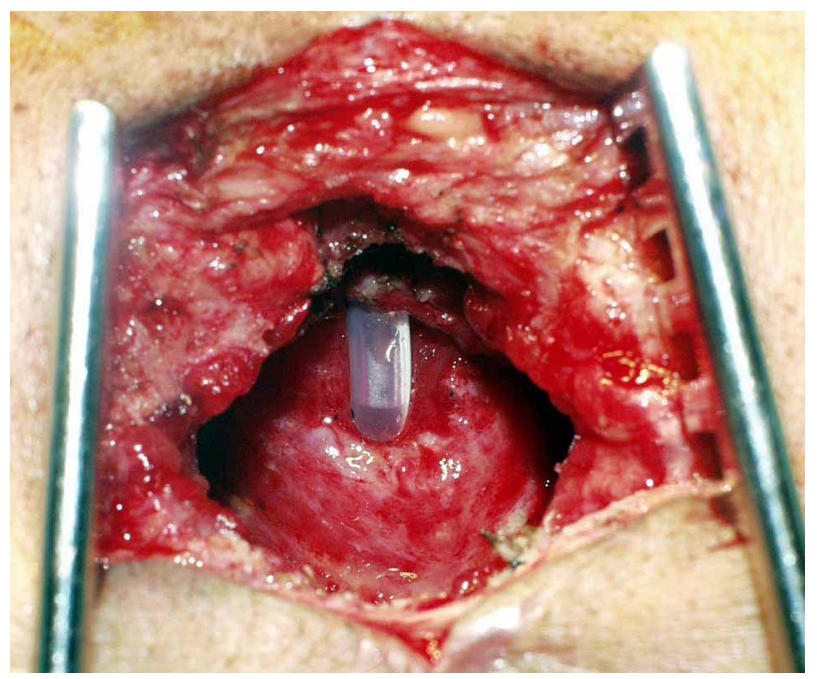

Figure 2. Perineal operative view shows the urethral defect measuring about $2.5 \mathrm{~cm}$ in diameter.

the balloon of the urethral catheter had become dislocated beyond the urethra. It was suspected that there had been bladder neck injury during the combined resection of the prostate and seminar vesicle, which allowed dislocation of the balloon. Another cause seemed to be that there was no supporting tissue behind the urinary system after the rectal resection. The dislodged balloon of the catheter might have caused rupture of the urethra at the thin and weak part after cancer resection.

Traumatic urethral injury is usually treated conservatively with an indwelling urethral catheter [1]. However,

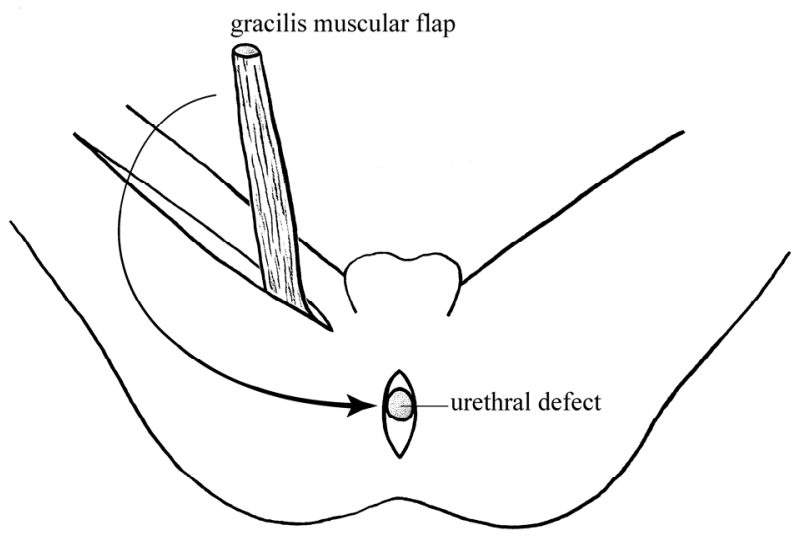

Figure 3. Two thirds of the muscular body of the gracilis muscle was separated from the proximal end. The muscular flap was transferred to the pelvic cavity through a subcutaneous route. The urethral defect was closed with the middle part of the flap. The pelvic cavity was filled with the distal part of the flap.

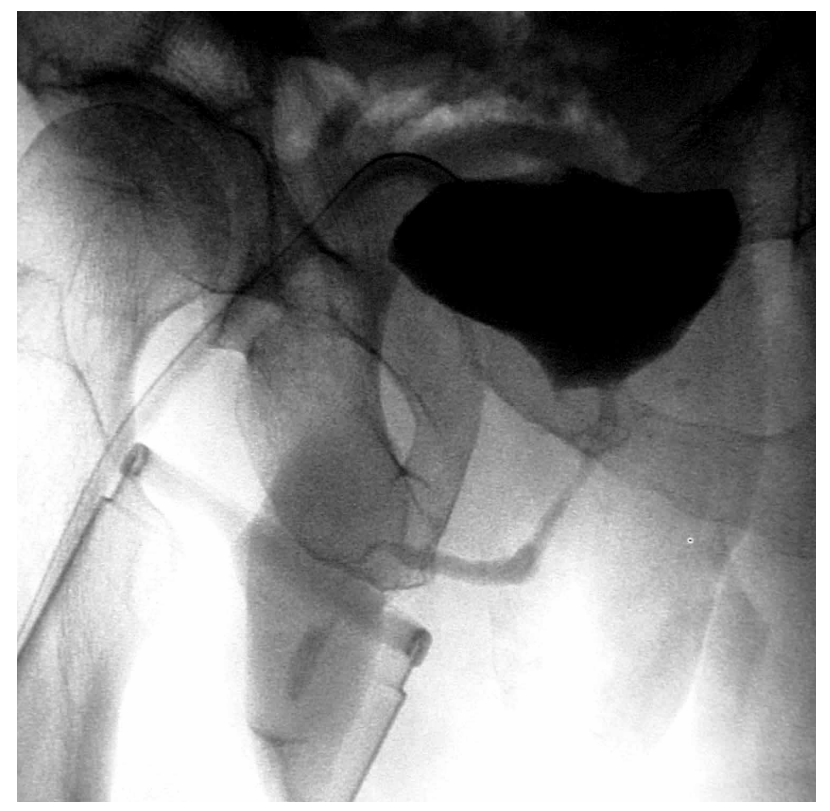

Figure 4. Postoperative cystography via vesicostomy shows no leakage.

after abdominoperineal resection of the rectum, healing of the prostatic urethra is apparently more difficult because there is no supporting tissue behind the urethra. Another disadvantage may have been the unstable placement of the balloon catheter in this case. The urethral defect seemed too large to approximate with a direct suture. Resection of the prostate seemed to be another choice for reconstruction of the urethra. However, the existence of dead space in the pelvis may introduce anastomotic failure and intractable urinary fistula.

There have been few reports describing the use of gracilis muscle to repair the urethra [2,3]. Beckenstein et al. [2] 
has reported the successful reconstruction of a circumferential defect of the urethra secondary to Fournier's gangrene using a gracilis flap. Lane et al. [3] has used a gracilis flap for radiotherapy induced recto-urethral fistula. It also have been used for reconstruction of the penis, scrotum and vagina [4,5]. Our experience with using a gracilis flap in several cases of perineal fistula or bed sore supported the choice of this procedure.

In the present case, good epithelization of the urethra was observed 5.5 years after surgery and there was no urethral stenosis. In other report, complete regeneration of the urethra has been noted following reconstruction using the garacilis [2]. The gracilis muscular flap is easily harvested with minimal impairment of gait [6]. We recommend this method for closure of urethral defect, when conservative therapy is unsuccessful.

\section{References}

[1] D. D. Morehouse, P. Belitsky and K. Mackinnon, "Rupture of the Posterior Urethra," Journal of Urology, Vol. 107, No. 2, 1972, pp. 255-258.
[2] M. Beckenstein, A. A. Smith, K. Dinchman, J. WyattAshmead and N. B. Meland, "Muscle Flap Reconstruction Aids in Urethral Regeneration," Annals of Plastic Surgery, Vol. 36, No. 6, 1996, pp. 641-643. doi:10.1097/00000637-199606000-00014

[3] B. R. Lane, D. E. Stein, F. H. Remzi, S. A. Strong, V. W. Fazio and K. W. Angermeier, "Management of Radiotherapy Induced Rectourethral Fistula,” Journal of Urology, Vol. 175, No. 4, 2006, pp. 1387-1388. doi:10.1016/S0022-5347(05)00687-7

[4] M. Orticochea, “A New Method of Total Reconstruction of the Penis,” British Journal of Plastic Surgery, Vol. 25, No. 4, 1972, pp. 347-366. doi:10.1016/S0007-1226(72)80077-8

[5] J. B. McCraw, F. M. Massey, K. D. Shanklin and C. E. Horton, "Vaginai Reconstruction with Gracilis Myocutaneous Flaps” Plastic and Reconstructive Surgery, Vol. 58, No. 2, 1976, pp. 176-183. doi:10.1097/00006534-197608000-00006

[6] R. H. Frederick, "Gracilis Myocutaneous and Muscle Flap,” Clinics in Plastic Surgery, Vol. 7, No. 1, 1980, pp. 27-44. 